\title{
ON CERTAIN SUFFICIENT CONDITIONS FOR THE CONVERGENCE AND CESĀRO SUMMABILITY OF THE ALLIED SERIES OF A DOUBLE FOURIER SERIES*
}

BY G. M. MERRIMAN $\dagger$

1. Introduction. The double Fourier series of a summable function

$$
\begin{aligned}
f(\alpha, \beta) & \sim \sum_{1}^{\infty} \sum_{1}^{\infty}\left\{\begin{array}{l}
a_{i, j} \cos i \alpha \cos j \beta+b_{i, j} \cos i \alpha \sin j \beta \\
+c_{i, j} \sin i \alpha \cos j \beta+d_{i, j} \sin i \alpha \sin j \beta
\end{array}\right\} \\
& =\sum_{1}^{\infty} \sum_{1}^{\infty}(a, b, c, d, \alpha, \beta)_{i, j}
\end{aligned}
$$

has three series said to be allied to it:

$$
\begin{aligned}
& \sum_{1}^{\infty} \sum_{1}^{\infty}(d,-c,-b, a, \alpha, \beta)_{i, j}, \\
& \sum_{1}^{\infty} \sum_{1}^{\infty}(c, d,-a,-b, \alpha, \beta)_{i, .} . \\
& \sum_{1}^{\infty} \sum_{1}^{\infty}(b,-a, d,-c, \alpha, \beta) i .
\end{aligned}
$$

These will be designated hereafter by the terms first, second, and third allied series, respectively.

Although the theorems concerning these series to be stated in this paper follow quite readily, in fact, so readily that most of the details of proof will be omitted, from the use of certain auxiliary functions and identities, yet they are useful and do not appear elsewhere in the literature. They were suggested by three papers of W. H. Young. $f$

* Presented to the Society, September 9, 1927.

$\dagger$ National Research Fellow.

$\ddagger$ (1) Multiple Fourier series, Proceedings of the London Society, (2), vol. 11 (1912-1913); (2) On the convergence of a Fourier series and its allied series, Proceedings of the London Society, (2), vol. 10 (1911-1912); (3) 
2. Convergence Theorems. If we write

$$
\begin{aligned}
& \phi_{1}(x, y) \equiv \frac{1}{4}\{f(\alpha+x, \beta+y)-f(\alpha+x, \beta-y)-f(\alpha-x, \beta+y)+f(\alpha-x, \beta-y)\} \\
& \sim \sum_{1}^{\infty} \sum_{1}^{\infty}(d,-c,-b, a, \alpha, \beta)_{i, j} \sin i x \sin j y, \\
& \phi_{2}(x, y) \equiv \frac{1}{4}\{f(\alpha+x, \beta+y)+f(\alpha+x, \beta-y) \\
&\quad-f(\alpha-x, \beta+y)-f(\alpha-x, \beta-y)\} \\
& \sim \sum_{1}^{\infty} \sum_{1}^{\infty}(c, d,-a,-b, \alpha, \beta)_{i, j} \sin i x \cos j y, \\
& \phi_{3}(x, y) \equiv \frac{1}{4}\{f(\alpha+x, \beta+y)-f(\alpha+x, \beta-y) \\
&\quad+f(\alpha-x, \beta+y)-f(\alpha-x, \beta-y)\} \\
& \sum_{1}^{\infty} \sum_{1}^{\infty}(b,-a, d,-c, \alpha, \beta)_{i, j} \cos i x \sin j y,
\end{aligned}
$$

we may state the following theorem.

THEOREM I. If $f(\alpha, \beta)$ is a function of bounded variation*, and if each of the integrals

$$
\begin{aligned}
& \lim _{\epsilon_{1}, \epsilon_{2} \rightarrow 0} \frac{1}{\pi^{2}} \int_{\epsilon_{1}}^{\pi} \int_{\epsilon_{2}}^{\pi} \phi_{1}(x, y) \operatorname{ctn}(x / 2) \operatorname{ctn}(y / 2) d x d y, \\
& \lim _{\epsilon_{1} \rightarrow 0} \frac{1}{\pi^{2}} \int_{\epsilon_{1}}^{\pi} \int_{0}^{\pi} \phi_{2}(x, y) \operatorname{ctn}(x / 2) d x d y, \\
& \lim _{\epsilon_{2} \rightarrow 0} \frac{1}{\pi^{2}} \int_{0}^{\pi} \int_{\epsilon_{2}}^{\pi} \phi_{3}(x, y) \operatorname{ctn}(y / 2) d x d y,
\end{aligned}
$$

Konvergenzbedingungen für die verwandte Reihen einer Fouriersche Reihe, Akademie der Wissenschaften München, 1911. (1) will hereafter be referred to as M.F.S., (2) as C.A.S., (3) as K.V.R.

* See M.F.S., §3; a function of two variables is said to have bounded variation if it is of bounded variation not only with respect to both variables, but also with respect to each variable separately. 
has a finite limit, then the three allied series of $f(\alpha, \beta)$ converge, (A) to (2), (B) and (C) to the negatives of (3) and (4), respectively.

Theorem $\mathrm{I}$ is a generalization of the result in K.V.R., \$2. By slight changes in the details of proof, we may obtain also two more general theorems, which will be needed later.

THEOREM II. If $\psi(u, v)$ is an odd-odd function of $u$ and $v$, and has the property that $u v \psi(u, v)$ is the double integral of some function $\phi(u, v)$ such that

$$
\int_{0}^{u} \int_{0}^{v}|\phi(u, v)| d u d v=o(u v),
$$

then the series of double Fourier constants of $\psi(u, v)$ (i.e., the first allied series of $\psi(u, v)$ at $u=0, v=0)$ converges to the value

$$
\lim _{\epsilon_{1}, \epsilon_{2} \rightarrow 0} \frac{1}{\pi^{2}} \int_{\epsilon_{1}}^{\pi} \int_{\epsilon_{2}}^{\pi} \psi(u, v) \operatorname{ctn}(u / 2) \operatorname{ctn}(v / 2) d u d v,
$$

provided that this limit exists.

THEOREM III. If $\psi(u, v)$ is an odd-even function of $u$ and $v$, such that $u \psi(u, v)$ is the integral with respect to $u$ of a function $\phi(u, v)$ such that

$$
\int_{0}^{u}|\phi(u, v)| d u=o(u)
$$

and $\phi(u, v)$ is, near $u=0, v=0$, a continuous function of $v$, and $a$ bounded function of $u$ and $v$, then the series of double Fourier constants of $\psi(u, v)$ converges to the limit

$$
\lim _{\epsilon_{1} \rightarrow 0} \frac{-1}{\pi^{2}} \int_{\epsilon_{1}}^{\pi} \int_{0}^{\pi} \psi(u, v) \operatorname{ctn}(u / 2) d u d v,
$$

if it exists.*

A theorem similar to Theorem III may be stated for an even-odd function.

${ }^{*}$ The continuity of $\psi(u, v)$ at the origin, essential to the proof of this theorem, follows from a result of $\mathrm{H}$. J. Ettlinger: On continuity in several variables, this Bulletin, vol. 33, No. 1 (Jan., 1927). 
3. Auxiliary Identities and Theorems. We write

$$
\begin{aligned}
& g_{1}(u, v)=\csc u \csc v \int_{0}^{u} \int_{0}^{v} \phi_{1}(2 u, 2 v) d u d v \\
& g_{2}(u, v)=\csc u \int_{0}^{u} \phi_{2}(2 u, 2 v) d u \\
& g_{3}(u, v)=\csc v \int_{0}^{v} \phi_{3}(2 u, 2 v) d v .
\end{aligned}
$$

If we carry out reductions akin to those in M.F.S., $\$ \$ 13,14$, and 21 , we derive the following set of identities :

(II) $\left[s_{C, C}\right]_{m, n}=4\left[S_{C, C}\right]_{m, n}-2\left[S_{C, O}\right]_{m, n}-2\left[S_{O, C}\right]_{m, n}+S_{m, n}$,

(III) $\left[s_{C, C}\right]_{m, n}=2\left[S_{C, C}\right]_{m, n}-\left[S_{O, C}\right]_{m, n}$,

(I V) $\left[s_{C . C}\right]_{m, n}=2\left[S_{C, C}\right]_{m, n}-\left[S_{C, O}\right]_{m, n}$.

In (I), $s_{m, n}$ stands for the partial sum of (A), $S_{m, n}$ for the partial sum of the double Fourier constants of $g_{1}(u, v), p_{m, n}$ for $m$ times the sum of the first $n$ terms of the $m$ th column of the double Fourier constants of $g_{1}(u, v), q_{m, n}$ for $n$ times the sum of the first $m$ terms of the $n$th row, and $A_{m, n}$ is the general term of the double Fourier constants of $g_{1}(u, v)$. In (II), the $s_{m, n}$ and $S_{m, n}$ stand for the same partial sums as in (I) and the subscripts $C$ and $O$ stand for "Cesàro" and "ordinary" summations respectively. In (III) and (IV), the $s_{m, n}$ is the partial sum of (B) and (C), the $S_{m, n}$ the partial sum of the double Fourier constants of $g_{2}(u, v)$ and $g_{3}(u, v)$, respectively. The last three identities can be extended to suitably defined Cesàro sums of higher indices. With these identities at hand, we may state the following results.

From repeated applications of (I) and (II), we obtain

THEOREM IV. If $g_{1}(u, v)$ be a summable function of $u$ and $v$ such that the functions $p_{m, n}, q_{m, n}$ and $m n A_{m, n}$ of its double Fourier constants have the unique double limit zero, then, if it be known that either the first allied series of $f(\alpha, \beta)$ or that of 
$g_{1}(u, v)$, at $u=0, v=0$, converges doubly in any Cesàro manner, indices $(r, r),{ }^{*}$ it follows that both series converge doubly in the ordinary manner and have the same sum.

COROLlARY. If the functions $p_{m, n}, q_{m, n}$ and $m n A_{m, n}$ converge to limits other than zero, the theorem is still true, except that the series then have not the same sum; the difference in their sums is the sum of the limits of the functions.

If we use (II) alone, and its extensions noted above, we have the following theorem.

TheOREM V. If the first allied series of the double Fourier series of $g_{1}(u, v)$ converges doubly in the ordinary way at the point $u=0, v=0$, then the first allied series of $f(\alpha, \beta)$ converges doubly in the Cesàro manner, indices $(1,1)$, and the sums of the series are the same.

This theorem can be extended to general Cesàro indices, and the footnote appended to Theorem IV shows that they need not be the same.

If we use (III) in the same manner, we obtain the theorem

THEOREM VI. If the second allied series of $g_{2}(u, v)$ converges doubly in the ordinary way at the point $u=0, v=0$, then the second allied series of $f(\alpha, \beta)$ converges $(C, 1,1)$, and the sums of the series are the same.

By use of (IV), a theorem similar to Theorem VI can be stated relative to the third allied series. Also, the theorems can be extended to higher Cesàro indices. Of course, we have not used the narrowest possible hypothesis by requiring the double convergence of the allied series of $g_{2}(u, v)$; we might have arrived at the same conclusions by requiring only the ordinary-Cesàro convergence. However, we shall need the result only as here stated.

We must note finally that we can replace the functions $g_{1}(u, v), g_{2}(u, v)$, and $g_{3}(u, v)$ by other auxiliary functions

* That these indices of summation may be different, say $(r, s)$, follows as in M.F.S., $\S \S 17,18$. 
$h_{1}(u, v), h_{2}(u, v)$, and $h_{3}(u, v)$, in which the factors csc $u$ and $\csc v$ have been replaced by $1 / u$ and $1 / v$ respectively. ${ }^{*}$

4. Summability $(C, 1,1)$. We proceed now to the main theorems of the paper.

THeOREM VII. If

$$
\int_{0}^{u} \int_{0}^{v}\left|\phi_{1}(2 u, 2 v)\right| d u d v=o(u v)
$$

and the limit

$$
\lim _{\epsilon_{1}, \epsilon_{2} \rightarrow 0} \frac{1}{\pi^{2}} \int_{\epsilon_{1}}^{\pi} \int_{\epsilon_{2}}^{\pi} \phi_{1}(u, v) \operatorname{ctn}(u / 2) \operatorname{ctn}(v / 2) d u d v
$$

exists, then the first allied series of $f(\alpha, \beta)$ sums $(C, 1,1)$ to (5).

The function $\phi_{1}(2 u, 2 v)$ fulfils the rôle of $\phi(u, v)$ in Theorem II, and therefore the first allied series of $h_{1}(u, v)$, and hence that of $g_{1}(u, v)$, converges at $u=0, v=0$, to the limit (if it exists)

$$
\lim _{\epsilon_{1}, \epsilon_{2} \rightarrow 0} \frac{1}{\pi^{2}} \int_{\epsilon_{1}}^{\pi} \int_{\epsilon_{2}}^{\pi} g_{1}(u, v) \operatorname{ctn}(u / 2) \operatorname{ctn}(v / 2) d u d v .
$$

Therefore, by Theorem $\mathrm{V}$, the first allied series of $f(\alpha, \beta)$ sums $(C, 1,1)$ to the value, if it exists,

$$
\begin{aligned}
\lim _{\epsilon_{1}, \epsilon_{2} \rightarrow 0} \frac{1}{4 \pi^{2}} \int_{\epsilon_{1}}^{\pi} \int_{\epsilon_{2}}^{\pi}\{ & \left.\int_{0}^{u} \int_{0}^{v} \phi_{1}(2 u, 2 v) d u d v\right\} \\
& \cdot \csc ^{2}(u / 2) \csc ^{2}(v / 2) d u d v .
\end{aligned}
$$

Integrating this result doubly by parts and using hypothesis, we complete Theorem VII.

Corollary. The limit (5) and the limit

$$
\lim _{\epsilon_{1}, \epsilon_{2} \rightarrow 0} \frac{1}{\pi^{2}} \int_{\epsilon_{1}}^{\infty} \int_{\epsilon_{2}}^{\infty} \frac{\phi_{1}(u, v)}{u v} d u d v
$$

are interchangeable, provided the periodicity of $f(\alpha, \beta)$ is used to extend its definition outside the given range.

* See C.A.S., $\S \S 5$ and 12. 
The expression (6) can be written in the form

$$
\lim _{\epsilon_{1}, \epsilon_{2} \rightarrow 0} \frac{4}{\pi^{2}} \int_{\epsilon_{1}}^{\infty} \int_{\epsilon_{2}}^{\infty} u^{-2} v^{-2}\left\{\int_{0}^{u} \int_{0}^{v} \phi_{1}(2 u, 2 v) d u d v\right\} d u d v
$$

whence, in tegrating doubly by parts and using the hypothesis, the corollary is proved.

Theorem VIII. If

$$
\int_{0}^{u} \int_{0}^{v}\left|\phi_{2}(2 u, 2 v)\right| d u d v=o(u v)
$$

and the limit

$$
\lim _{\epsilon_{1} \rightarrow 0} \frac{-1}{\pi^{2}} \int_{\epsilon_{1}}^{\pi} \int_{0}^{\pi} \phi_{2}(u, v) \operatorname{ctn}(u / 2) d u d v
$$

exists, then the second allied series of $f(\alpha, \beta)$ sums $(C, 1,1)$ to (8).

The function $\phi_{2}(2 u, 2 v)$ is easily seen to play the part of $\phi(u, v)$ in Theorem III, and hence the second allied series of $h_{2}(u, v)$ and therefore that of $g_{2}(u, v)$ converge to the limit (if it exists)

$$
\lim _{\epsilon_{1} \rightarrow 0} \frac{-1}{\pi^{2}} \int_{\epsilon_{1}}^{\pi} \int_{0}^{\pi} g_{2}(u, v) \operatorname{ctn}(u / 2) d u d v .
$$

Hence, by Theorem VI, the second allied series of $f(\alpha, \beta)$ sums $(C, 1,1)$ to

$$
\lim _{\epsilon_{1} \rightarrow 0} \frac{-1}{2 \pi^{2}} \int_{\epsilon_{1}}^{\pi} \int_{0}^{\pi}\left\{\int_{0}^{u} \phi_{2}(2 u, 2 v) d u\right\} \csc ^{2}(u / 2) d u d v .
$$

An integration by parts with respect to $u$, and the use of hypothesis, complete Theorem VIII.

A theorem similar to Theorem VIII can be deduced concerning the third allied series of $f(\alpha, \beta)$.

In conclusion, mention should, be made perhaps, of theorems analogous to those of $\S \S 3$ and 4 , dealing with summability $(C, r, 0)$ and $(C, 0, s)$ of the allied series, which can be developed along the lines of this paper from functions 
of the type of $g_{2}(u, v)$ and $g_{3}(u, v)$. It would be such theorems which would allow us to arrive at the statements contained in the footnote appended to Theorem IV and elsewhere.

In the proofs of Theorems $\mathrm{V}$ and VI, use was made of a "consistency theorem" to the effect that if a double series is summable $(C, r, s)$, it is also summable $\left(C, r^{\prime}, s^{\prime}\right), r^{\prime} \geqq r, s^{\prime} \geqq s$, and to the same sum as formerly. This result is contained in Theorem I' of a paper by the author concerning the summability of double series of a certain type, which will appear in an early issue of the Annals of Mathematics.

HARVARD UNIVERSITY

\section{A GENERAL THEOREM ON QUANTIC DETERMINANTS*}

BY T. R. ROSEBRUGH

Let there be a homogeneous linear substitution

$$
x_{r j}^{\prime}=\sum_{i=1}^{n_{r}} a_{r i i} x_{r i}, \quad\left(j=1,2, \cdots, n_{r}\right),
$$

having $M_{r}$ for the determinant of its coefficients, and let there be derived therefrom quantics of degree $p_{r}$ giving values of all possible monomials in these $n_{r}$ new variables of that degree. There will be

$$
f_{r}=\left(\begin{array}{c}
p_{r}+n_{r}-1 \\
p_{r}
\end{array}\right)
$$

such new monomials, and an equal number of the old will be involved in these quantics.

Now consider this to be but one, namely the $r$ th of a set of $s$ such substitutions, each in its own set of variables of arbitrary (generally different) number $n_{r}$, similarly treated. Each new monomial of each of the $s$ sets is now to be used,

* Presented to the Society, February 26, 1927. 